\title{
Effect of competition between petroleum-degrading bacteria and indigenous compost microorganisms on the efficiency of petroleum sludge bioremediation: field application of mineral-based culture in the composting process
}

\author{
Hamid Abtahi ${ }^{\mathrm{a}}$, Milad Parhamfar ${ }^{\mathrm{b}}$, Reza Saeedi ${ }^{\mathrm{c}, \mathrm{d}}$, José Villaseñor ${ }^{\mathrm{e}}$, Majid Sartaj ${ }^{\mathrm{f}}$, Vinod \\ Kumar $^{\mathrm{g}}$, Frederic Coulong, Maryam Parhamfar ${ }^{\mathrm{a}}$, Mojtaba Didehdar ${ }^{\mathrm{h}}$, Hamed seifi ${ }^{\mathrm{i}}$, Ali \\ Koolivand $^{\mathrm{j} *}$
}

a Molecular and Medicine Research Center, Arak University of Medical Sciences, Arak, Iran

b Faculty of Science, Department of Chemistry, Duissburg-Essen University, Essen, Germany

c Workplace Health Promotion Research Center, Shahid Beheshti University of Medical Sciences, Tehran, Iran

d Department of Health, Safety and Environment (HSE), School of Public Health and Safety, Shahid Beheshti University of Medical Sciences,

Tehran, Iran

e Department of Chemical Engineering, Institute of Chemical \& Environmental Technologies, University of Castilla-La Mancha, Campus

Universitario S/n,13071, Ciudad Real, Spain

f University of Ottawa, Department of Civil Engineering, 161 Louis Pasteur, Ottawa, Ontario K1N 6N5, Canada

g School of Water, Energy and Environment, Cranfield University, Cranfield, United Kingdom

h Department of Medical Parasitology and Mycology, Arak University of Medical Sciences, Arak, Iran

i Department of Electrical and Computer Engineering, West Tehran Branch, Islamic Azad University, Tehran, Iran

j Department of Environmental Health Engineering, Faculty of Health, Arak University of Medical Sciences, Arak, Iran

*Corresponding Author: Ali Koolivand, Tel: +988633686443; Fax:+988633686443; Email address: alikoulivand@arakmu.ac.ir

\section{Abbreviations list ${ }^{1}$}

${ }^{1} \mathrm{BH}$, Bushnel-Haas; $\mathrm{C}_{\text {org }}$, organic carbon; FC, finished compost ; ICM, indigenous compost microorganisms; MATH, microbial adhesion to hydrocarbon; MBC, mineral-based culture; OD, optical density; PDB, petroleum degrading bacteria; PHCs, petroleum hydrocarbons; PWS, petroleum waste sludge; TPHs, total petroleum hydrocarbons 


\begin{abstract}
The effect of competition between isolated petroleum-degrading bacteria (PDB) and indigenous compost microorganisms (ICM) on the efficiency of composting process in bioremediation of petroleum waste sludge (PWS) was investigated. After isolating two native PDB (Acinetobacter radioresistens strain KA5 and Enterobacter hormaechei strain KA6) from PWS, their ability for growth and crude oil degradation was examined in the mineral-based culture (MBC). Then, the PDB isolate were inoculated into the composting experiments and operated for 12 weeks. The results showed that the PDB degraded $21.65-68.73 \%$ of crude oil (1$5 \%$ ) in the MBC after 7 days. The PDB removed $84.30 \%$ of total petroleum hydrocarbon (TPHs) in the composting bioreactor containing the initial TPH level of $20 \mathrm{~g} \mathrm{~kg}^{-1}$. Removal of petroleum hydrocarbons (PHCs) in the composting experiments proceeded according to the first-order kinetics. The computed values of degradation rate constants and half-lives showed a better performance of the PDB than ICM for TPHs removal. This finding suggests that simultaneous application of the PDB and ICM in the composting reactors resulted in a decline in the effectiveness of the PDB which is due to competition between them. The study also verified that the capability of PDB in degrading PHCs can be successfully scaled-up from MBC to composting process.
\end{abstract}

Keywords: Petroleum waste sludge; Petroleum-degrading bacteria; Indigenous compost microorganisms; Bioremediation 


\section{Introduction}

Petroleum hydrocarbons (PHCs) contamination is still posing significant environmental and human health concerns around the world due to the release of petroleum waste sludge (PWS) generated from oil refinery plants (Robichaud et al., 2019; Siles and Margesin, 2018). The complex constituents of PWS, including PHCs, ammonia, sulfides, etc., make conventional treatment methods inefficient to meet national and international environmental regulations (Srikanth et al., 2018). Over the years, different physical and chemical strategies have been used, with varying efficacy, to solve the environmental issues of PHCs (Chen et al., 2019). The existing treatment methods suffer from a number of problems such as such as high energy requirement, processes complexity, and incomplete contaminant removal which has led to the search for alternative sustainable treatment technologies (Srikanth et al., 2018; Varjani, 2017). Bioremediation which relies on the use of biological processes has been recognized as an economical, efficient, and environmentally friendly approach to treat petroleum-contaminated environments (Awasthi et al., 2018; Huang et al., 2019). From this point of view, composting process has been frequently deemed for treating PHCs (Ren et al., 2018).

The application of hydrocarbon-degrading bacteria as a consortium could improve the degradation efficacy and promote the bioremediation process (Awasthi et al., 2018; Varjani, 2017). Over the years, several petroleum-degrading bacteria (PDB) from PWS have been isolated, which are able to grow rapidly and degrade various concentrations of PHCs (Awasthi et al., 2018; Demichelis et al., 2017). Although bioremediation of PWS has been greatly advanced in recent years, there are still some challenges when the developed bioremediation technology under laboratory conditions is implemented under real environmental conditions. Thus, one of the most important challenges of bioremediation is the low potential of field application of 
culture-based experiments. This may be attributed to unfavorable environmental conditions in the field and competition from other indigenous microorganisms in natural environments such as compost (Awasthi et al., 2018; Li et al., 2019b; Tao et al., 2019). Numerous studies have reported that PDB isolated from contaminated environments, exhibit decreased enzymatic activities as the time progresses because of unfavorable conditions (Awasthi et al., 2018; Demichelis et al., 2017). Sayara et al. (2011) reported that the inoculation of the fungus $T$. versicolor did not increase remediation efficiency since indigenous microorganisms were probably better adapted to the environment. Since the effectiveness of bioremediation is dependent on the survival and activity of the inoculated consortia mainly affected by the competition of PDB with indigenous microorganisms, debate around the benefits of bacterial inoculation and its capacity to increase the microbial degradation of PHCs continues (Jiang et al., 2016; Tao et al., 2019).

Therefore, it is necessary to pay attention to the survival and competition of PDB with the indigenous microorganisms present in petroleum-contaminated environments. Considering this background, the novelty of this work lies in the investigation of the competition between exogenous PDB and indigenous compost microorganisms (ICM) during the field application of a mineral-based culture (MBC) in a composting process for bioremediation of PWS. Hence, this study was performed to: (i) isolate PDB with high degradation potential for PHCs and bioaugmentation; (ii) investigate the possible competition between the inoculated consortia and ICM during PWS composting; and (iii) assess the field application of MBC in PWS bioremediation through bioaugmentated composting process.

\section{Materials and methods}




\subsection{Collection and preparation of the PWS and finished compost (FC) Samples}

The PWS, collected from an oil refinery plant in Iran, had the following basic characteristics (dry weight basis): TPHs value of $255.05 \mathrm{~g} \mathrm{~kg}^{-1}$, organic carbon $\left(\mathrm{C}_{\mathrm{org}}\right)$ level of $528.96 \mathrm{~g} \mathrm{~kg}^{-1}$, moisture content of $27.63 \%$, $\mathrm{pH}$ of 6.10 , total nitrogen content of $1.75 \mathrm{~g} \mathrm{~kg}^{-1}$, and total phosphorus content of $1.03 \mathrm{~g} \mathrm{~kg}^{-1}$. The FC used as a bulking agent was purchased from a local company and passed through a 2-mm sieve to remove sands and other impurities. The initial properties of the FC were as follows (dry weight basis): TPHs background level of $0.79 \mathrm{~g} \mathrm{~kg}^{-1}$,

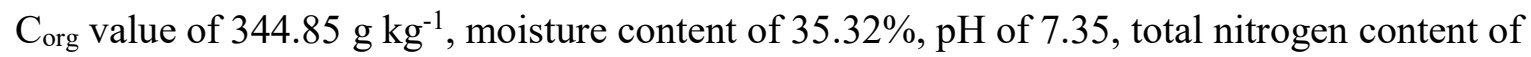
$4.25 \mathrm{~g} \mathrm{~kg}^{-1}$, and total phosphorus content of $2.78 \mathrm{~g} \mathrm{~kg}^{-1}$. The PWS and FC samples were stored in a dark at room temperature before the start of the composting experiments. Some samples of the PWS and $\mathrm{FC}$ were steam sterilized at $121^{\circ} \mathrm{C}$ for $2 \mathrm{~h}$ to eliminate any existing indigenous microorganisms.

\subsection{Isolation and identification of the $P D B$}

The PDB used in this research were isolated from the PWS. For PDB isolation, $5 \mathrm{~g}$ of the PWS was mixed with $100 \mathrm{ml}$ of mineral Bushnell-Haas (BH) medium. Crude oil (1\%) was also added to the medium to be used as a source of energy and carbon. After shaking for 1 week at 30 ${ }^{\circ} \mathrm{C}$, fresh medium was inoculated with $5 \mathrm{ml}$ of the incubated culture. Following repeating this enrichment procedure for several times, $100 \mu 1$ of the medium was spread over the nutrient agar surface and incubated. Finally, a total of 24 strains were isolated from the enrichment tests. In order to examine the growth rate and degradation abilities of all the isolated strains, they were added to the mixture of $\mathrm{BH}$ and $1 \%$ crude oil. Cell growth was determined using optical density (OD) at $600 \mathrm{~nm}$ after 2, 4, 7, 10, and 12 days. 
A number of tests including citrate, urease, catalase, oxidase, triple sugar iron, $\mathrm{H}_{2} \mathrm{~S}$ production, nitrate reduction, indole production, and gram staining were conducted for the identification of the strains (Table 1). Molecular identification was performed by the 16S rRNA gene amplification and sequencing according to the standard procedure available in the previous papers (Koolivand et al., 2019b; Poorsoleiman et al., 2019).

\subsection{Optimization of culture-based experiments}

\subsubsection{Impact of $p H$}

To study the effect of $\mathrm{pH}$ on crude oil degradation by the PDB, experiments were conducted at different $\mathrm{pH}$ values including $4,5,6,7,8$, and $9 . \mathrm{HCl}$ and $\mathrm{NaOH}$ were used to adjust $\mathrm{pH}$ to the appropriate level. The bacterial mixture ( $0.5 \mathrm{McFarland})$ was added to $\mathrm{BH}$ medium with crude oil $(1 \%)$ and incubated for 7 days at $30^{\circ} \mathrm{C}$. After the incubation period, both the growth rate and crude oil biodegradation were measured. The biodegradation of crude oil was measured on the basis of the removal rate of TPHs in the culture-based experiments. The removal rates of TPHs were determined as the TPHs removal against the control experiments containing no bacterial addition. The TPHs removal rate was calculated as follows:

TPHs removal rate $=[($ initial TPHs - final TPHs $) /$ initial TPHs $] \times 100$

where the initial TPHs and final TPHs are the amounts of TPHs before and after treatment, respectively.

\subsubsection{Impact of initial TPHs concentration}

The strains mixture was exposed to the incremental concentrations of crude oil ranging from 1 to $5 \%$ so as to determine the highest levels of biodegradation by the PDB. The above-mentioned 
amounts of crude oil were mixed with BH solution of $500 \mathrm{ml}$ in the Erlenmeyer flasks. The PDB $\left(1.5 \times 10^{8} \mathrm{CFU} / \mathrm{ml}\right)$ was added to them (at initial $\mathrm{pH}$ of 7$)$ and incubated at $30{ }^{\circ} \mathrm{C}$. After an incubation period of 7 days at $120 \mathrm{rpm}$, the TPHs degradation was measured. The crude oil concentration exhibiting the maximum TPHs removal was selected as the optimized level to be used in the composting bioreactors. It is worth pointing out that the control tests with no bacterial addition were also conducted, under the same conditions, in all the above mentioned culturebased experiments.

\subsubsection{Measurement of microbial adhesion to hydrocarbon (MATH)}

Determination of MATH was performed through cell surface hydrophobicity as described by Chen et al. (2018) with slight modifications. Briefly, after adding the PDB to nutrient agar and incubating for $24 \mathrm{~h}$ at $30^{\circ} \mathrm{C}$, one colony was mixed with a buffer solution and the initial concentration of microbial cell was measured in the prepared suspensions. Then, in a clean glass tube, $5 \mathrm{~mL}$ of the bacterial suspension was added to $200 \mu \mathrm{l}$ of hexadecane. The tube was stirred by vortex ( $2 \mathrm{~min})$ and set aside (30 $\mathrm{min})$ for phase separation. By using a Pasteur pipette, a sample of the suspension was retrieved when the hydrocarbon droplet size was stable. Next, the cell concentration of the retrieved bacterial sample was determined. The MATH was calculated based on the following equation:

$$
\text { MATH }(\%)=\left[1-\left(\mathrm{OD}_{1} / \mathrm{OD}_{2}\right)\right] \times 100
$$

where $\mathrm{OD}_{1}$ and $\mathrm{OD}_{2}$ are the concentrations of the bacterial suspension before and after mixing, respectively.

\subsubsection{Measurement of emulsification activity $\left(E_{24}\right)$}


Emulsification activity was conducted using the bacterial culture medium and cell-free supernatant according to the procedure published previously (Bayat et al., 2016; Patowary et al., 2017). Briefly, the PDB were blended with nutrient broth, incubated and then the cell-free supernatant was obtained from the cultures centrifugation. After centrifugation, the cell-free supernatant and culture medium were mixed with crude oil of $1 \%$ concentration and vortexed vigorously for $2 \mathrm{~min}$. The solution was allowed to stand $(24 \mathrm{~h})$ at room temperature. The $\mathrm{E}_{24}$ was measured using the following equation:

$$
\mathrm{E}_{24}(\%)=\left(\mathrm{H}_{\mathrm{e}} / \mathrm{H}_{\mathrm{t}}\right) \times 100
$$

where $\mathrm{H}_{\mathrm{e}}(\mathrm{cm})$ is the emulsified height, and $\mathrm{H}_{\mathrm{t}}(\mathrm{cm})$ is the total mixture height. $\mathrm{E}_{24}$ was expressed as the percentage of the overall height occupied by the emulsion.

\subsection{Composting experiments}

\subsubsection{Experimental design of the bioreactors}

Composting experiments were carried out in the chemical resistant polypropylene containers to evaluate the removal efficiency of the process. Each composting container (5-L capacity) contained $3 \mathrm{Kg}$ of a mixture of PWS and FC. Four conditions were tested as follows:

$\mathrm{D}_{1}$ : autoclaved PWS + autoclaved FC with no PDB inoculation as a control experiment to determine abiotic loss of TPHs

$\mathrm{D}_{2}$ : autoclaved PWS + autoclaved FC + PDB inoculation to evaluate the PDB potential for TPHs degradation

$\mathrm{D}_{3}$ : autoclaved PWS + non-autoclaved FC to investigate the ICM potential for TPHs degradation 
$\mathrm{D}_{4}$ : autoclaved PWS + non-autoclaved FC + PDB inoculation for measurement of the combined potential of the PDB and ICM for TPHs degradation to determine the PDB and ICM competition

The initial TPH concentrations in the composting reactors were adjusted at $20 \mathrm{~g} \mathrm{~kg}^{-1}$ based on the findings of the bioremediation tests performed in the mineral-based experiments (section 2.3.2). All the experiments were conducted in triplicate.

\subsubsection{Operation of the bioreactors}

The mixture of the PWS and FC was thoroughly blended in an attempt to minimize the heterogeneous distribution of TPHs. Each reactor was turned over several times to allow good aeration over the 12-week duration of the process. To enhance aeration and guarantee sufficient oxygen, aeration (at $11 \mathrm{~min}^{-1} \mathrm{~kg}^{-1}$ ) was provided by oil-free pumps (Koolivand et al., 2019c). The nitrogen and phosphorus were balanced to an optimum C/N/P ratio of 100/5/1 (Koolivand et al., 2018). During the composting phase, watering was conducted twice a week to maintain the moisture content in the range of $45-55 \%$. The water was taken from the city of Arak's municipal supply. The reactors $\mathrm{D}_{2}$ and $\mathrm{D}_{4}$ were designed for the PDB inoculation with an initial concentration of approximately $1.5 \times 10^{8} \mathrm{CFU} \mathrm{g}^{-1}$ dry mixture.

\subsubsection{Sampling procedure}

Seven samples were collected at predetermined intervals $(0,2,4,6,8,10$, and 12 weeks). For each sampling event, three sub-samples were taken from various depths, blended and homogenized into one composite sample for each bioreactor. The homogenized samples were placed in a glass jar and kept at dark and room temperature until analysis. 


\subsection{Quantification methods}

TPHs in the samples was determined following the TNRCC (2001) method. First, 5 gram of composting sample was weighed and dried at $105^{\circ} \mathrm{C}$. Then, $5 \mathrm{ml}$ of n-pentane was added, mixed well, and preserved until the time of analysis. After extraction of PHCs content in the samples, a gas chromatography equipped with a flame ionization detector (GC-FID) (Shimadzu, Japan) was used for quantification of TPHs. The detailed description of the GC-FID method is available in a paper by Koolivand et al. (2013a). After shaking the mixture of composting samples ( $1 \mathrm{~g})$ with distilled water $(10 \mathrm{~mL})$ for $30 \mathrm{~min}, \mathrm{pH}$ was determined on the aqueous extract (Khadra et al., 2019; Tmecc, 2002). $\mathrm{C}_{\mathrm{org}}$ was determined by loss on incineration according to the method of TMECC (2002). Moisture readings were taken gravimetrically by oven drying at $105^{\circ} \mathrm{C}$. All the chemicals used in this study were of analytical grade and bought from Sigma-Aldrich Company.

\subsection{Data analysis}

In the present study, the statistical analyses were performed using Microsoft Excel 2013 and SPSS package (version 11.0). All the tests were performed in triplicate to get reliable data. The obtained results were indicated as means \pm standard deviations on the basis of dry weight. Linear regression analysis was applied to show the relation between the variables. Chromas software was used to analyze the sequencing results. The sequences were aligned with the CLUSTAL X 2.0. Similarity searches of the sequences compared to NCBI database were done using BLAST. The phylogenetic trees were drawn using MEGA 7 software.

\section{Results and discussion}

\subsection{Taxonomic and biochemical identification of the PDB}


The results of NCBI search indicated that the isolated PDB are Acinetobacter radioresistens strain KA5 and Enterobacter hormaechei strain KA6. The phylogenetic trees for the two strains are shown in Fig. 1 and Fig. 2. The sequences of the strains KA5 and KA6 have been deposited in GenBank (NCBI) under the accession numbers MK127547 and MK127548, respectively. Table 1 also presents the results of the biochemical tests conducted for them.

Fig. 1

Fig. 2

Table 1

\subsection{Culture-based experiments}

\subsubsection{Metabolic properties of the PDB}

The capability of the PDB for growth and crude oil consumption in the MBC was examined. The $\mathrm{OD}_{600}$ of $0.42,1.12,1.67,1.75$, and 1.49 were obtained after the incubation time of $2,4,7$, 10, and 12 days, respectively. It can be deduced from these values that the growth logarithmic phase of the PDB lasted 7-10 days. For this reason, the 7-day incubation duration was selected for all the experiments performed in the MBC. The values of MATH and $\mathrm{E}_{24}$ were calculated to be 23.78 and $31.18 \%$, respectively, showing the possibility of the PDB affinity to PHCs and biosurfactant production.

\subsubsection{Impact of $\mathrm{pH}$ on crude oil removal}

It is necessary to pay attention to $\mathrm{pH}$ as a basic parameter for bacterial growth and activity. Any fluctuations in $\mathrm{pH}$ can change the solubility and degradation of hydrocarbons ( $\mathrm{Li}$ et al., 2019a; Muangchinda et al., 2018). Hence, in the current work, the dependency of bacterial growth and crude oil degradation to $\mathrm{pH}$ in the $\mathrm{MBC}$ was evaluated. This helps to find the best $\mathrm{pH}$ 
and perform the composting experiment at the optimized $\mathrm{pH}$. The obtained results (Table 2) showed that the PDB had the highest rate $(67.46 \%)$ of crude oil degradation at $\mathrm{pH} 7$. The growth rate of the PDB and bioremediation rate slightly declined at pHs of 6 and 8 , while the removal efficiency dropped dramatically to the values of 43.21 and $45.90 \%$ respectively in the $\mathrm{pH}$ values 5 and 9. These observations are in line with the results reported by Muangchinda et al. (2018). It has also been reported that the maximum bacterial growth and metabolic activity occurred in the appropriate $\mathrm{pH}$ range of 6-8 (Li et al., 2019a; Ma et al., 2016). By using these MBC results for scaling-up bioremediation process, we operated the composting experiments under the neutral $\mathrm{pH}$.

\section{Table 2}

\subsubsection{Impact of crude oil concentration}

The optimization of TPHs level is one of the most determining factors affecting pollutants degradation during bioremediation process. Thereupon, the effect of crude oil concentration was also investigated to scale-up the PDB ability for decomposing PHCs from MBC to composting process. As can be inferred from Table 3, the PDB grew well in BH medium in the presence of $1-5 \%$ crude oil. The degradation rates were computed to be $67.46,68.73,62.88,37.54$, and $21.65 \%$ respectively for the oil amounts of $1,2,3,4$, and $5 \%$ after the incubation period of 7 days. It comes to no surprise that the removal efficiency went down with the higher initial concentration as the huge amounts of petroleum compounds are toxic to the bacteria. However, in the case of $2 \%$ crude oil, the bacterial growth as well as biodegradation effectiveness was higher than that of $1 \%$. The possible reason for this behavior is that a very low concentration of PHCs is not detectable and thereby bacterial activity and bioremediation efficiency are limited (Vaidya et al., 2017; Varjani and Upasani, 2017). Accordingly, it can be stated that the initial 
content of crude oil is of utmost importance in performing bioremediation strategies. The findings resulted from the MBC experiments demonstrated that $2 \%$ concentration of oil was the optimal level in which the PDB exhibited the highest degradation rate.

Table 3

Two reasons may account for the potential of the PDB for growth and mineralization of the wide range of oil concentrations (1-5\%). First, it is because of the inherent metabolic capabilities of the strains KA5 and KA6 isolated from the PWS. The second reason can be attributed to the fact that the synergistic effects between these two different strains makes the overall process more efficient. Since PWS has been made from various hydrocarbons, and each strain metabolizes specific compounds, application of strains mixture yields better results (Kamyabi et al., 2017; Mnif et al., 2015).

Tables 2 and 3 also indicated that the higher biomass production $\left(\mathrm{OD}_{600} \mathrm{~nm}\right)$ of the PDB leads to more biodegradation of crude oil. The regression analysis provided in Fig. 3 showed a strong correlation between biomass production and oil degradation exhibited by the PDB.

Fig. 3

\subsection{Field application of the $M B C$ in the composting process}

\subsection{Degradation of the PWS using biological composting experiments}

As numerous factors can impact the TPHs-removal achieved in a bioremediation system, field studies may yield different results than laboratory ones (Robichaud et al., 2019). In order to examine the possibility of field PHCs bioremediation using bioaugmentation, the potential of the PDB in degrading the PWS was determined in the composting treatments. The experimental study of the PWS degradation in the composting reactors was done based on the MBC results. Accordingly, the primary TPHs content in the bioreactors was $20 \mathrm{~g} \mathrm{~kg}^{-1}$ through adjusting the 
mixing ratios of the PWS to the FC. Adjustment of this mixing ratio as an effective variable of composting process is of importance since it impacts the degradation rate of the target pollutants and also the process cost (Farzadkia et al., 2019; Koolivand et al., 2013b).

The variations of TPHs concentration with time in each composting reactor have been shown in Fig. 4. After 12 weeks, TPHs content reduced from the initial level of $20 \mathrm{~g} \mathrm{~kg}^{-1}$ to $18.86,3.14$, 12.74 , and $5.12 \mathrm{~g} \mathrm{~kg}^{-1}$, respectively in the experiments $\mathrm{D}_{1}, \mathrm{D}_{2}, \mathrm{D}_{3}$, and $\mathrm{D}_{4}$. These amounts correspond to the total TPHs removal of $5.70,84.30,36.30$, and $74.4 \%$, respectively. The very low level of removal in the control treatment $\left(\mathrm{D}_{1}\right)$ demonstrated that TPHs dissipation is mainly due to the metabolic activities of the microbial community present in the composting experiments. Accordingly, the abiotic mechanisms such as volatilization were negligible and were not responsible for the remarkable reduction of TPHs.

In the present study, the variation of TPHs content in the composting experiments $\mathrm{D}_{2}$, and $\mathrm{D}_{4}$ containing the PDB showed an almost similar decreasing trend with time. It can be observed that the most rapid TPHs biodegradation was obtained during the initial 8 weeks of composting, followed by a gradual reduction of biodecomposition rate over the remaining composting time. It has been also reported from the previous works (Koolivand et al., 2019a; Koolivand et al., 2014) that degradation of petroleum compounds during composting proceeds rapidly in the beginning weeks of the process and slows down in the later. A possible reason for this trend is that easily accessible and biodegradable hydrocarbons which can be dissolved in water phase or can be absorbed by the microbial community are degraded first, while the remaining fractions are difficult to degradation (Ren et al., 2018; Robichaud et al., 2019). Furthermore, the generation of petroleum metabolites such as naphthenic acids and fatty acids during the process can limit 
further biodegradation due to the suppressed degradative activities of the microbial population (Chen et al., 2019; Pacwa-Płociniczak et al., 2019).

\section{Fig. 4}

\subsubsection{Competition between the PDB and ICM}

The percentage of TPHs degradation observed in the treatments $\mathrm{D}_{3}(36.30 \%)$ indicated limited capabilities of the ICM for biodegradation. In such cases when indigenous degradingmicroorganisms are not efficient enough, it is useful to introduce active PDB to enhance the remediation efficacy. As the treatment $\mathrm{D}_{2}$ was found to have the highest TPHs removal, the high ability of the PDB for biodecomposition of PHCs was verified. Hence, the PDB inoculation was introduced into the treatment $\mathrm{D}_{4}$ containing the ICM in order to enhance degradation capacity. Although the overall biodegradation efficacy of the reactor $\mathrm{D}_{4}$ increased due to the PDB addition, it was still lower than that of the $\mathrm{D}_{2}$. The possible reason may be attributed to the competition between the PDB and ICM. This means that not all inoculated microorganisms are able to be active in contaminated environments due to the competition of the native with nonnative microorganisms for growth and consuming the available sources of carbon and energy. The native species of compost may also act as a barrier to the activities of inoculated nonnative species due to the fact that the inoculated microorganisms may be preyed by some intrinsic species such as protozoa. Moreover, the metabolite of the ICM can inhibit the activity of the PDB since the major populations observed in the mixed culture may be involved in the antagonistic interactions between microbes (Liang et al., 2018; Ren et al., 2018). There are some studies reporting no significant difference in TPHs removal for the experiments with addition of petroleum-degrading microorganisms. Thion et al. (2012) observed antagonistic interactions 
between the mixed culture of microorganisms in bioremediation of contaminated soils. Tao et al. (2019) also reported competition between exogenous inoculated and indigenous microorganisms. Table 4 provides the percentages of TPHs removal in each sampling week. A very important point to make about this Table is that the removal efficiency of the experiment $\mathrm{D}_{4}$ is not improved greatly until the week 4 which is due to the PDB adaptation and their competition with the ICM. During the week 4-8, the degradation and removal of TPHs enhanced considerably. During this period, the percentage of TPHs removal occurred in the reactor $\mathrm{D}_{4}$ was by far higher than that of the reactor $\mathrm{D}_{3}$. This indicated that the PDB isolated from the PSW can effectively survive and adapt to this environment containing the ICM only at this period. Again, the decrease in the ability of the PDB to degrade TPHs over the last 4 weeks of the study could be attributed to the recalcitrant nature of the remaining PHCs. Besides, possible nutrient deficiency and lower levels of the residual TPHs can lead to increased competition between the PDB and ICM.

\section{Table 4}

The performed regression analysis of data plotted in Fig. 4 showed that the PHCs biodegradation can be regarded as the first-order kinetics. The biodegradation rate constants calculated for the experiments $\mathrm{D}_{2}, \mathrm{D}_{3}$, and $\mathrm{D}_{4}$ were $0.160,0.042$, and $0.132 \mathrm{~d}^{-1}$, respectively. The corresponding half-lives were found to be $4.33,16.50$, and 5.25 days, respectively. The firstorder rate constants reported by He et al. (2019) and Gomez and Sartaj (2013) were respectively in the range of $0.0003-0.0049$ and $0.004-0.043 \mathrm{~d}^{-1}$. The reason for these differences is due to the fact that the rate constant is dependent on multiple factors such as pollutants nature and concentration, bioremediation method, and characteristics of the microbial population (He et al., 2014; Kulikowska, 2016). The higher biodegradation constant and lower half-life observed in the 
reactor $\mathrm{D}_{2}$ compared to those in the experiment $\mathrm{D}_{3}$ indicated that the PDB are more efficient than the ICM when being used alone. However, the comparisons of these values with those observed in the reactor $\mathrm{D}_{4}$ verified the competition between them. In the future, more studies should be directed at competition mechanisms of the inoculated strains with the intrinsic populations.

\subsubsection{Competitive impact of FC addition}

In the composting of PWS, co-substrates such as FC are added to provide sufficient readily degradable $\mathrm{C}_{\text {org. }}$. These materials also provide microbial communities and slow-release nutrients that can support the degradation of target pollutants (Chen et al., 2019). Additionally, it acts as a physical support for composting environment, reduces the toxicity of PHCs, and adjust the moisture and aeration of the process so as to promote microbial activity and thereby contributes to effective TPHs degradation (Kästner and Miltner, 2016; Robichaud et al., 2019). However, since microorganisms have a more tendency to readily degradable $\mathrm{C}_{\text {org }}$ than the resistant petroleum compounds, the excess addition of these materials may compete with the microbial metabolism of PHCs. Therefore, a proper content of FC should be taken into account to balance the competing effect with PWS and motivating effect on microorganisms (Ren et al., 2018). In order to clarify the competing or motivating effect of the $\mathrm{FC}$ addition, the evolution of $\mathrm{C}_{\mathrm{org}} / \mathrm{TPHs}$ were presented in Fig. 5a. The increment pattern of $\mathrm{C}_{\text {org }} / \mathrm{TPHs}$ observed in the reactors $\mathrm{D}_{2}$ and $\mathrm{D}_{4}$ was an indication of the higher metabolization of PHCs than that of $\mathrm{C}_{\text {org }}$. This demonstrated that the FC contents of the composting reactors did not exhibit competing effect with the PWS. It was reported from the two field experiments conducted by Gomez and Sartaj (2013) and Robichaud et al. (2019) that the addition of compost accelerated the removal of target contaminants over the same time span (3 months) of the present study. 
Fig. $5 \mathrm{~b}$ showed that the trend of $\mathrm{C}_{\text {org }}$ change is similar to that of TPHs. Since the base

371

372

373
Fig. 5

\section{Conclusions}

The field application of the MBC in the composting process as well as the competition of the PDB with the ICM during PWS bioremediation was investigated. The isolated PDB were capable of degrading crude oil and PWS in the MBC and composting process. The decline in the PDB efficiency, when inoculated into the ICM containing reactor, indicated a competition between the PDB and ICM. However, in the presence of the ICM, the PDB could effectively metabolize TPHs at the weeks 4-8 of the composting time.

\section{Acknowledgements}

The authors thankfully acknowledge the financial support from Arak University of Medical Sciences (Grant No. 2819). We also thank Mr. Vahid Koolivand for providing the PWS. 
Awasthi, M.K., Selvam, A., Chan, M.T., Wong, J.W.C. 2018. Bio-degradation of oily food waste employing thermophilic bacterial strains. Bioresour. Technol. 248, 141-147.

Bayat, Z., Hassanshahian, M., Hesni, M.A. 2016. Study the symbiotic crude oil-degrading bacteria in the mussel Mactra stultorum collected from the Persian Gulf. Mar. Pollut. Bullet. 105(1), 120-124.

Chen, F., Li, X., Zhu, Q., Ma, J., Hou, H., Zhang, S. 2019. Bioremediation of petroleumcontaminated soil enhanced by aged refuse. Chemosphere 222, 98-105.

Chen, X., Song, D., Xu, J., Li, E., Sun, G., Xu, M. 2018. Role and mechanism of cell-surface hydrophobicity in the adaptation of Sphingobium hydrophobicum to electronic-waste contaminated sediment. Appl. Microbiol. Biotechnol. 102(6), 2803-2815.

Demichelis, F., Pleissner, D., Fiore, S., Mariano, S., Navarro Gutiérrez, I.M., Schneider, R., Venus, J. 2017. Investigation of food waste valorization through sequential lactic acid fermentative production and anaerobic digestion of fermentation residues. Bioresour. Technol. 241, 508-516.

Farzadkia, M., Esrafili, A., Gholami, M., Koolivand, A. 2019. Effect of immature and mature compost addition on petroleum contaminated soils composting: kinetics. J. Environ. Health Sci. Eng. Doi: 10.1007/s40201-019-00400y.

Gomez, F., Sartaj, M. 2013. Field scale ex-situ bioremediation of petroleum contaminated soil under cold climate conditions. Int. Biodeter. Biodegrad. 85, 375-382.

He, J., Fan, X., Liu, H., He, X., Wang, Q., Liu, Y., Wei, H., Wang, B. 2019. The study on Suaeda heteroptera Kitag, Nereis succinea and bacteria's joint bioremediation of oilcontaminated soil. Microchem. J. 147, 872-878. 
He, X.S., Xi, B.D., Zhang, Z.Y., Gao, R.T., Tan, W.B., Cui, D.Y. 2014. Insight into the evolution, redox, and metal binding properties of dissolved organic matter from municipal solid wastes using two-dimensional correlation spectroscopy. Chemosphere 117, 701-707.

Huang, Y., Pan, H., Wang, Q., Ge, Y., Liu, W., Christie, P. 2019. Enrichment of the soil microbial community in the bioremediation of a petroleum-contaminated soil amended with rice straw or sawdust. Chemosphere 224, 265-271.

Jiang, Y., Brassington, K.J., Prpich, G., Paton, G.I., Semple, K.T., Pollard, S.J., Coulon, F. 2016. Insights into the biodegradation of weathered hydrocarbons in contaminated soils by bioaugmentation and nutrient stimulation. Chemosphere 161, 300-307.

Kamyabi, A., Nouri, H., Moghimi, H. 2017. Synergistic effect of sarocladium sp. and cryptococcus sp. co-culture on crude oil biodegradation and biosurfactant production. Appl. Biochem. Biotechnol. 182(1), 324-334.

Kästner, M., Miltner, A. 2016. Application of compost for effective bioremediation of organic contaminants and pollutants in soil. Appl. Microbiol. Biotechnol. 100(8), 3433-3449.

Khadra, A., Ezzariai, A., Merlina, G., Capdeville, M.J., Budzinski, H., Hamdi, H., Pinelli, E., Hafidi, M. 2019. Fate of antibiotics present in a primary sludge of WWTP during their cocomposting with palm wastes. Waste Manage. 84, 13-19.

Koolivand, A., Abtahi, H., Godini, K., Saeedi, R., Rajaei, M.S., Parhamfar, M. 2019a. Biodegradation of oil tank bottom sludge using a new two-phase composting process: kinetics and effect of different bulking agents. J. Mater. Cycles Waste Manage. 21(6), $1280-1290$.

Koolivand, A., Abtahi, H., Parhamfar, M., Didehdar, M., Saeedi, R., Fahimirad, S. 2019b. Biodegradation of high concentrations of petroleum compounds by using indigenous 
bacteria isolated from petroleum hydrocarbons-rich sludge: Effective scale-up from liquid medium to composting process. J. Environ. Manage. 248, 109228.

438

Koolivand, A., Godini, K., Saeedi, R., Abtahi, H., Ghamari, F. 2019c. Oily sludge biodegradation using a new two-phase composting method: Kinetics studies and effect of aeration rate and mode. Process Biochem. 79, 127-134.

Koolivand, A., Naddafi, K., Nabizadeh, R., Jafari, A., Nasseri, S., Yunesian, M., Yaghmaeian, K., Alimohammadi, M. 2014. Application of hydrogen peroxide and fenton as pre-and post-treatment steps for composting of bottom sludge from crude oil storage tanks. Pet. Sci. Technol. 32(13), 1562-1568.

Koolivand, A., Naddafi, K., Nabizadeh, R., Nasseri, S., Jafari, A.J., Yunesian, M., Yaghmaeian, K. 2013a. Degradation of petroleum hydrocarbons from bottom sludge of crude oil storage tanks using in-vessel composting followed by oxidation with hydrogen peroxide and Fenton. J. Mater. Cycles Waste Manage. 15(3), 321-327.

Koolivand, A., Naddafi, K., Nabizadeh, R., Nasseri, S., Jafari, A.J., Yunesian, M., Yaghmaeian, K., Nazmara, S. 2013b. Biodegradation of petroleum hydrocarbons of bottom sludge from crude oil storage tanks by in-vessel composting. Toxicol. Environ. Chem. 95(1), 101-109.

Koolivand, A., Naddafi, K., Nabizadeh, R., Saeedi, R. 2018. Optimization of combined in-vessel composting process and chemical oxidation for remediation of bottom sludge of crude oil storage tanks. Environ. Technol. 39(20), 2597-2603.

Kulikowska, D. 2016. Kinetics of organic matter removal and humification progress during sewage sludge composting. Waste Manage. 49, 196-203. 
Li, R., Munoz, G., Liu, Y., Sauvé, S., Ghoshal, S., Liu, J. 2019a. Transformation of novel polyfluoroalkyl substances (PFASs) as co-contaminants during biopile remediation of petroleum hydrocarbons. J. Hazard. Mater. 362, 140-147.

Li, X., Zheng, R., Zhang, X., Liu, Z., Zhu, R., Zhang, X., Gao, D. 2019b. A novel exoelectrogen from microbial fuel cell: Bioremediation of marine petroleum hydrocarbon pollutants. J. Environ. Manage. 235, 70-76.

Liang, J., Cheng, T., Huang, Y., Liu, J. 2018. Petroleum degradation by Pseudomonas sp. ZS1 is impeded in the presence of antagonist Alcaligenes sp. CT10. AMB Express 8(1), 88.

Ma, J., Yang, Y., Dai, X., Chen, Y., Deng, H., Zhou, H., Guo, S., Yan, G. 2016. Effects of adding bulking agent, inorganic nutrient and microbial inocula on biopile treatment for oilfield drilling waste. Chemosphere 150, 17-23.

Mnif, I., Mnif, S., Sahnoun, R., Maktouf, S., Ayedi, Y., Ellouze-Chaabouni, S., Ghribi, D. 2015. Biodegradation of diesel oil by a novel microbial consortium: comparison between coinoculation with biosurfactant-producing strain and exogenously added biosurfactants. Environ. Sci. . Pollut. Res. 22(19), 14852-14861.

Muangchinda, C., Rungsihiranrut, A., Prombutara, P., Soonglerdsongpha, S., Pinyakong, O. 2018. 16S metagenomic analysis reveals adaptability of a mixed-PAH-degrading consortium isolated from crude oil-contaminated seawater to changing environmental conditions. J. Hazard. Mater. 357, 119-127.

Pacwa-Płociniczak, M., Czapla, J., Płociniczak, T., Piotrowska-Seget, Z. 2019. The effect of bioaugmentation of petroleum-contaminated soil with Rhodococcus erythropolis strains on removal of petroleum from soil. Ecotoxicol. Environ. Saf. 169, 615-622. 
Patowary, K., Patowary, R., Kalita, M.C., Deka, S. 2017. Characterization of biosurfactant produced during degradation of hydrocarbons using crude oil as sole source of carbon. Front. Microbiol. 8, 279.

Poorsoleiman, M.S., Hosseini, S.A., Etminan, A., Abtahi, H., Koolivand, A. 2019. Effect of twostep bioaugmentation of an indigenous bacterial strain isolated from oily waste sludge on petroleum hydrocarbons biodegradation: Scaling-up from a liquid mineral medium to a two-stage composting process. Environ. Technol. Innov. 100558.

Ren, X., Zeng, G., Tang, L., Wang, J., Wan, J., Wang, J., Deng, Y., Liu, Y., Peng, B. 2018. The potential impact on the biodegradation of organic pollutants from composting technology for soil remediation. Waste Manage. 72, 138-149.

Robichaud, K., Girard, C., Dagher, D., Stewart, K., Labrecque, M., Hijri, M., Amyot, M. 2019. Local fungi, willow and municipal compost effectively remediate petroleum-contaminated soil in the Canadian North. Chemosphere 220, 47-55.

Sayara, T., Borràs, E., Caminal, G., Sarrà, M., Sánchez, A. 2011. Bioremediation of PAHscontaminated soil through composting: influence of bioaugmentation and biostimulation on contaminant biodegradation. Int. Biodeter. Biodegrad. 65(6), 859-865.

Siles, J.A., Margesin, R. 2018. Insights into microbial communities mediating the bioremediation of hydrocarbon-contaminated soil from an Alpine former military site. Appl. Microbiol. Biotechnol. 102(10), 4409-4421.

Srikanth, S., Kumar, M., Puri, S.K. 2018. Bio-electrochemical system (BES) as an innovative approach for sustainable waste management in petroleum industry. Bioresour. Technol. $265,506-518$. 
Tao, K., Zhang, X., Chen, X., Liu, X., Hu, X., Yuan, X. 2019. Response of soil bacterial community to bioaugmentation with a plant residue-immobilized bacterial consortium for crude oil removal. Chemosphere 222, 831-838.

Thion, C., Cébron, A., Beguiristain, T., Leyval, C. 2012. PAH biotransformation and sorption by Fusarium solani and Arthrobacter oxydans isolated from a polluted soil in axenic cultures and mixed co-cultures. Int. Biodeter. Biodegrad. 68, 28-35.

TMECC. 2002. Test methods for the examination of composting and compost. USDA and US Composting Council.

TNRCC. 2001. Total petroleum hydrocarbons, method 1005. Texas Natural Resource Conservation Commission.

Vaidya, S., Jain, K., Madamwar, D. 2017. Metabolism of pyrene through phthalic acid pathway by enriched bacterial consortium composed of Pseudomonas, Burkholderia, and Rhodococcus (PBR). 3 Biotech 7(1), 29.

Varjani, S.J. 2017. Microbial degradation of petroleum hydrocarbons. Bioresour. Technol. 223, 277-286.

Varjani, S.J., Upasani, V.N. 2017. A new look on factors affecting microbial degradation of petroleum hydrocarbon pollutants. Int. Biodeter. Biodegrad. 120, 71-83. 
Table 1. Biochemical identifications of the two bacterial strains isolated from the petroleum waste sludge

Table 2. Effect of $\mathrm{pH}$ (at crude oil concentration of $1 \%$ ) on the biodegradation rate of crude oil in mineral-based culture after a period of 7 days

Table 3. Effect of initial crude oil concentration (at the $\mathrm{pH}$ value of 7) on the biodegradation rate of crude oil in mineral-based culture after a period of 7 days

Table 4. Percentages of TPHs removal in the composting reactors over the process duration

\section{Figure captions}

Fig. 1. Phylogenetic tree based on $16 \mathrm{~S}$ rRNA gene sequences of Acinetobacter radioresistens strain KA5 isolated from the petroleum waste sludge

Fig. 2. Phylogenetic tree based on 16S rRNA gene sequences of Enterobacter hormaechei strain KA6 isolated from the petroleum waste sludge

Fig. 3. Correlation between bacterial growth and crude oil degradation in the mineral-based culture

Fig. 4. Trend of TPHs degradation in the composting reactors over the process duration $\left(\mathrm{D}_{1}\right.$ : autoclaved PWS + autoclaved FC with no PDB inoculation as a control experiment; $\mathrm{D}_{2}$ : autoclaved PWS + autoclaved FC + PDB inoculation; $\mathrm{D}_{3}$ : autoclaved PWS + nonautoclaved FC; D4: autoclaved PWS + non-autoclaved FC + PDB inoculation)

Fig. 5. Trend of (a) $\mathrm{C}_{\mathrm{org}} / \mathrm{TPH}$ and (b) $\mathrm{C}_{\text {org }}$ changes in the composting reactors and (c) correlation between TPHs and $\mathrm{C}_{\text {org }}$ over the process duration $\left(\mathrm{D}_{1}\right.$ : autoclaved PWS + autoclaved FC with no PDB inoculation as a control experiment; $\mathrm{D}_{2}$ : autoclaved PWS + autoclaved FC + 
PDB inoculation; $\mathrm{D}_{3}$ : autoclaved PWS + non-autoclaved FC; $\mathrm{D}_{4}$ : autoclaved PWS + non-

547 autoclaved FC + PDB inoculation)

548 


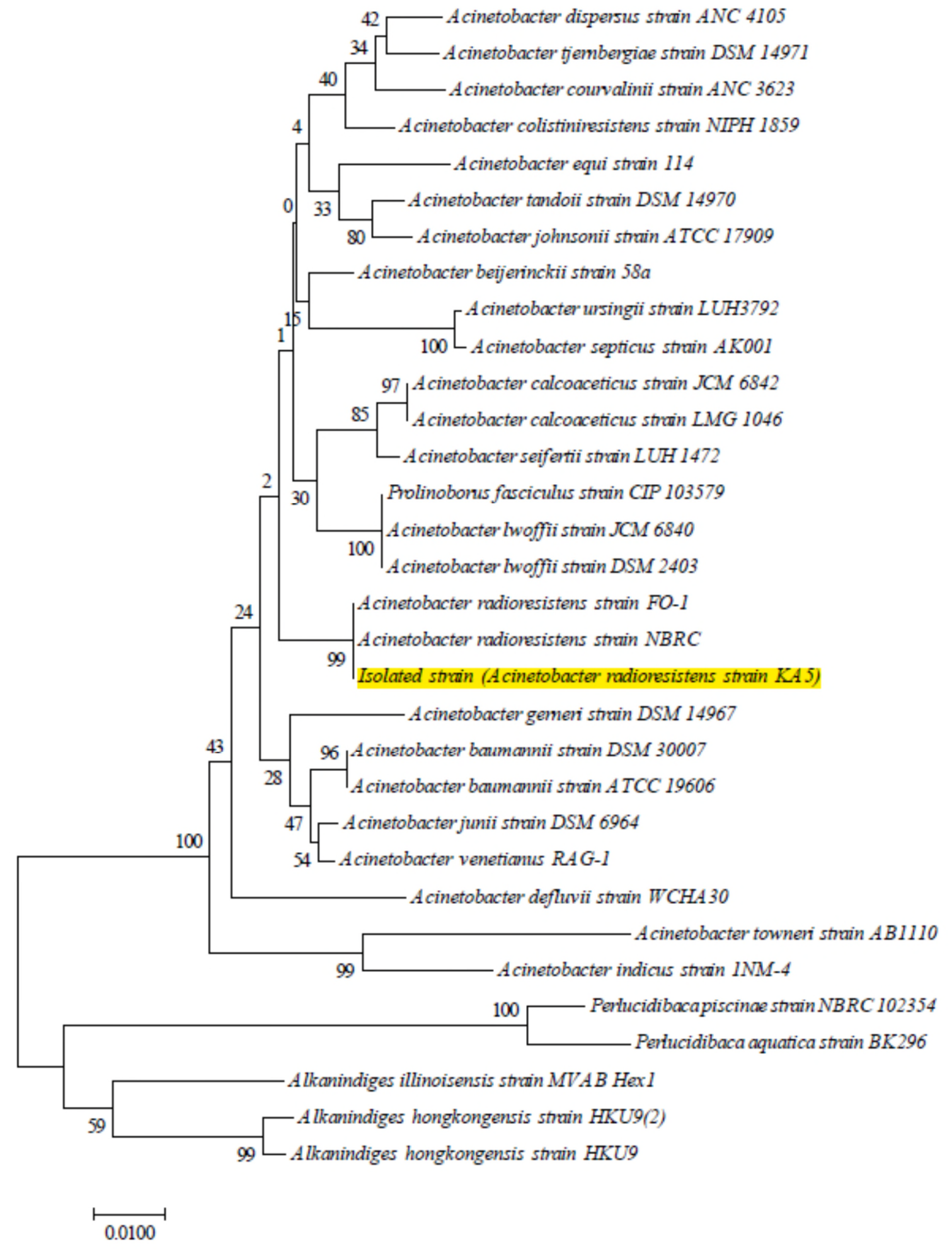

550

551 


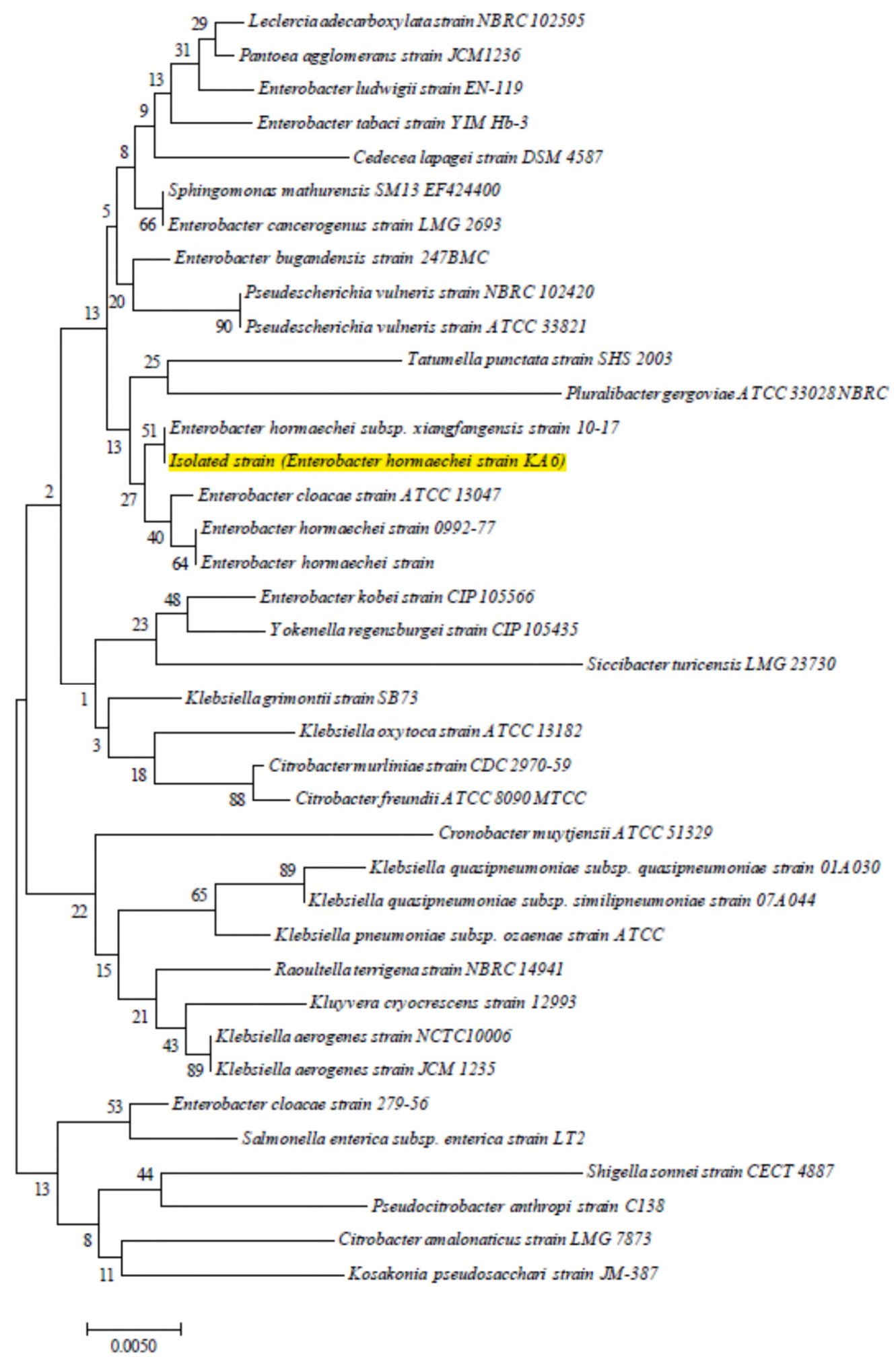

552

Figure 2 


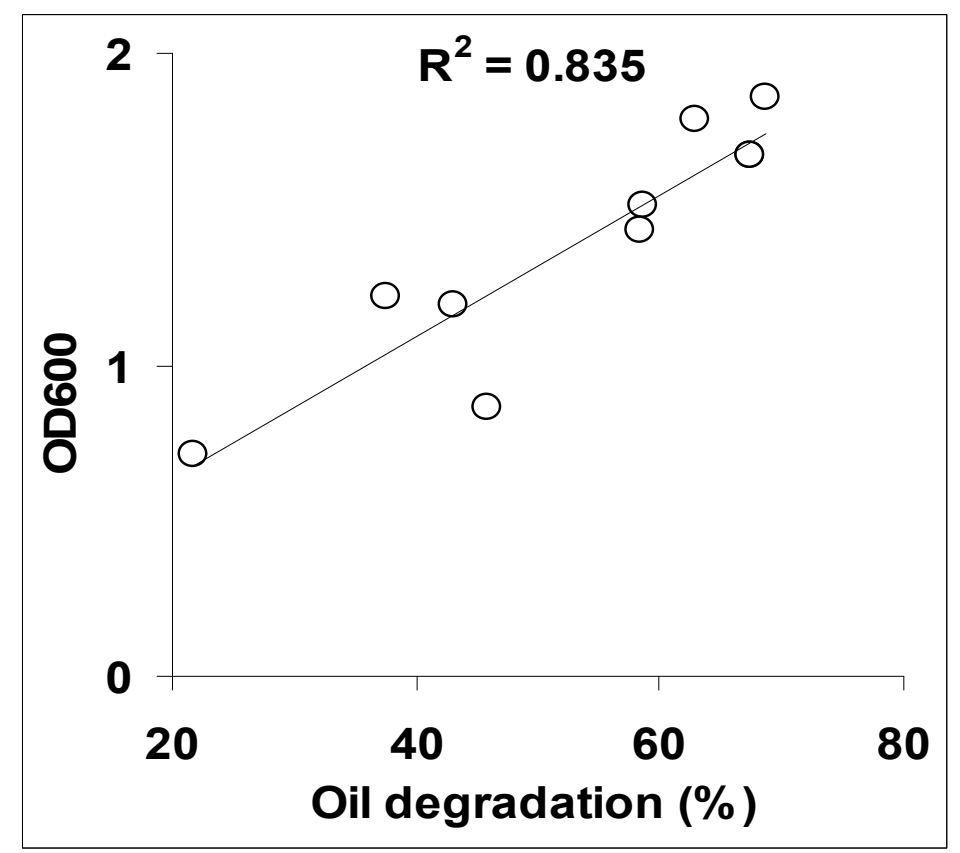

556 Figure 3

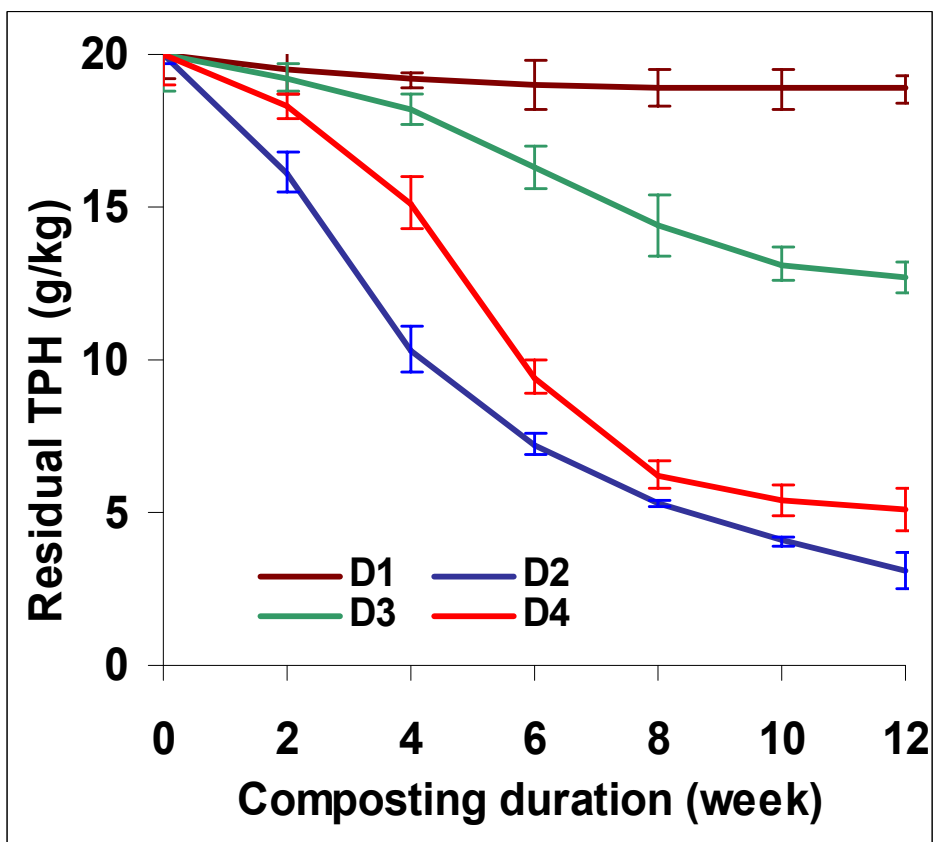

559 Figure 4 

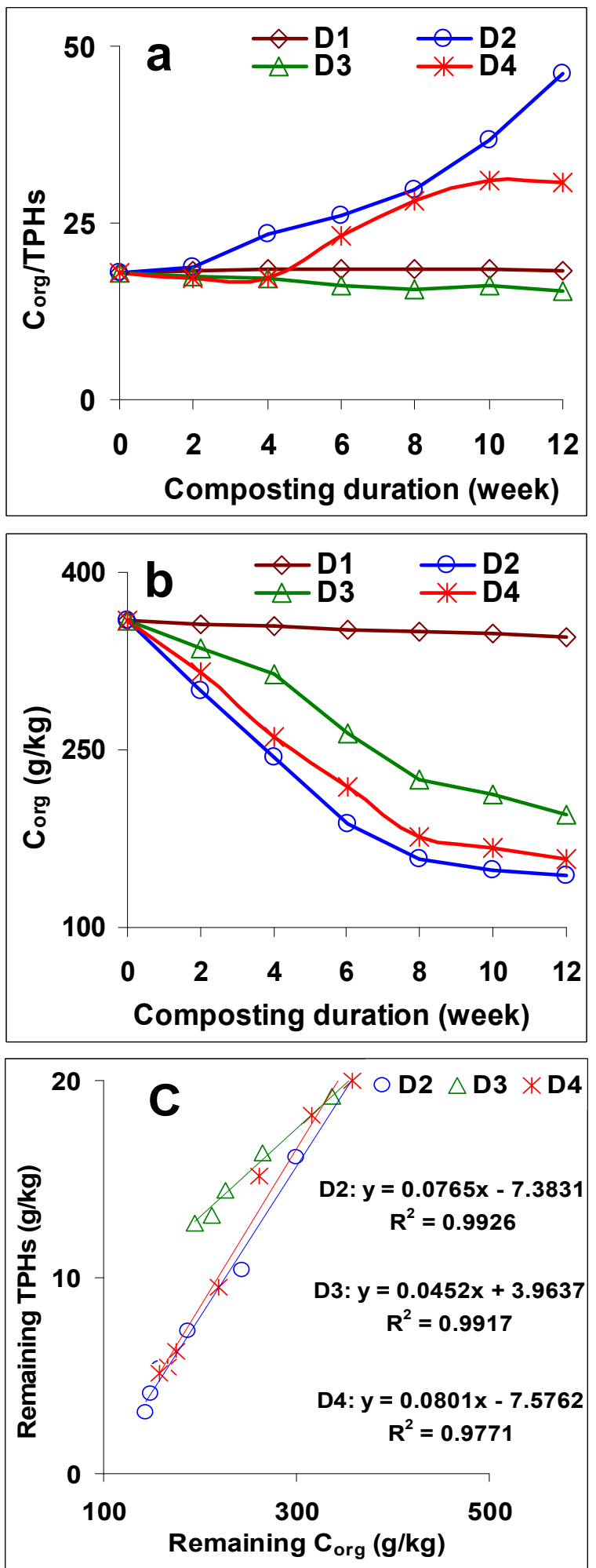

Figure 5 
Table 1

\begin{tabular}{lll}
\hline \multicolumn{1}{c}{ Tests } & $\begin{array}{l}\text { Acinetobacter } \\
\text { radioresistens } \\
\text { Strain KA5 }\end{array}$ & $\begin{array}{l}\text { Enterobacter } \\
\text { hormaechei Strain } \\
\text { KA6 }\end{array}$ \\
\hline Gram stain & Gram negative & Gram negative \\
Oxidase & - & - \\
Catalase & + & + \\
Nitrate reduction & - & + \\
Citrate & - & + \\
Urease & - & - \\
H 2 S production & - & - \\
Indole production & - & - \\
Triple sugar iron & Alkaline/Alkaline & Acid/Acid \\
\hline
\end{tabular}

563

564 Table 2

565

\begin{tabular}{lll}
\hline pH & OD 600 & $\begin{array}{l}\text { Crude oil } \\
\text { removal (\%) }\end{array}$ \\
\hline 5 & 1.19 & 43.21 \\
6 & 1.51 & 58.67 \\
7 & 1.67 & 67.46 \\
8 & 1.43 & 58.48 \\
9 & 0.86 & 45.90 \\
\hline
\end{tabular}

566 Table 3

\begin{tabular}{lll}
\hline $\begin{array}{l}\text { Crude oil } \\
\text { concentrations (\%) }\end{array}$ & OD $_{600}$ & $\begin{array}{l}\text { Crude oil } \\
\text { removal (\%) }\end{array}$ \\
\hline 1 & 1.67 & 67.46 \\
2 & 1.86 & 68.73 \\
3 & 1.79 & 62.88 \\
4 & 1.22 & 37.54 \\
5 & 0.71 & 21.65 \\
\hline
\end{tabular}


Table 4

\begin{tabular}{lllll}
\hline $\begin{array}{l}\text { Process time } \\
\text { (week) }\end{array}$ & \multicolumn{3}{l}{ TPH removal rate (\%) } & \\
\cline { 2 - 5 } & $\mathbf{D}_{\mathbf{1}}{ }^{*}$ & $\mathbf{D}_{2}{ }^{* *}$ & $\mathbf{D}_{\mathbf{3}}{ }^{* * *}$ & $\mathbf{D}_{\mathbf{4}}{ }^{* * * *}$ \\
\hline 0 & 0.00 & 0.00 & 0.00 & 0.00 \\
2 & 2.25 & 19.40 & 3.95 & 8.60 \\
4 & 1.90 & 28.85 & 5.05 & 15.70 \\
6 & 0.75 & 15.55 & 9.40 & 28.45 \\
8 & 0.40 & 9.65 & 9.60 & 16.10 \\
10 & 0.35 & 6.25 & 6.30 & 4.15 \\
12 & 0.05 & 4.60 & 2.00 & 1.40 \\
Total & 5.70 & 84.30 & 36.30 & 74.40 \\
\hline
\end{tabular}

569

* autoclaved PWS + autoclaved FC with no PDB inoculation as a control experiment

570

** autoclaved PWS + autoclaved FC + PDB inoculation

571

*** autoclaved PWS + non-autoclaved FC

572

**** autoclaved PWS + non-autoclaved FC + PDB inoculation

573 